\title{
Dynamic surface tension measured with an integrated sensor-actuator device using electrolytically generated gas bubbles
}

\author{
Wouter Olthuis*, Alex Volanschi** and Piet Bergveld* \\ *MESA Research Institute, University of Twente \\ P.O. Box 217, 7500 AE Enschede, The Netherlands \\ **Unilever Research Laboratory Vlaardingen \\ P.O. Box 114, 3130 AC Vlaardingen, The Netherlands
}

\section{SUMMARY}

In this paper, a new, simple method to determine dynamic surface tension in aqueous solutions is reported, explained and experimentally verified [1]. By function integration, a small device is obtained; apart from control and interface electronics no external components or systems are necessary. Instead of the conventional sparging, we use the (cathodic) electrolysis of water at an actuator to produce gas bubbles in situ. The actuator can also function as the sensor for the bubble frequency by monitoring either its overpotential or its impedance.

Keywords: Dynamic surface tension, Stirfactants, Electrolytically generated gas bubbles.

\section{INTRODUCTION}

The dynamic surface tension (DST) of an aqueous solution determines its wetting and cleaning ability, e.g., in a washing process. Within the range of interest for washing, the DST is monotonously related to the concentration of surfactants, forming an important part of the formulation of washing powder. The DST decreases for increasing surfactant concentration.

Conventional methods of DST determination are based on the investigation of phenomena occurring at the new interfaces produced by blowing gas bubbles (sparging) in a solution containing surfactants. One method is the determination of the maximum bubble pressure [2]. The fluctuating pressure in the bubble during growth reaches a maximum value, related to the DST and the capillary radius of the sparging device. Another

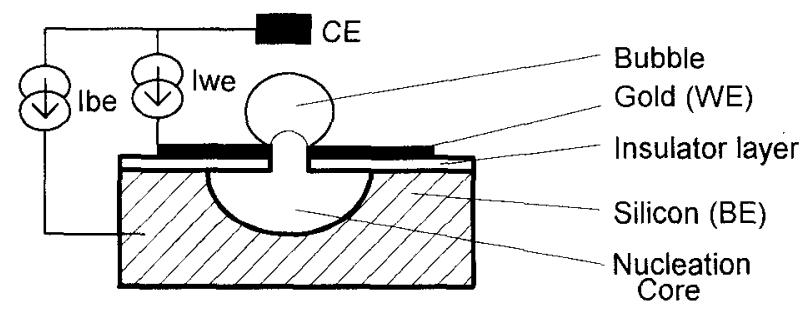

Fig. 1 Cross-sectional view of a gas phase nucleation core (GPNC) electrode with one nucleation site. $I_{\text {we }}$ is the electrolysis current used to generate bubbles at the working electrode WE and $I_{b e}$ is the current used for the generation of the gas phase nucleation core at the bulk electrode BE. method is that of the pressure-jump [3], measuring the frequency of bubble formation and subsequent release from the sparging capillary. At a constant gas flow, this frequency turns out to be related to the DST. Both methods require a separate sensing unit for the pressure measurement or the frequency determination and, moreover, some kind of gas compressor and supply unit. These units cause the conventional measuring systems to be relatively large and unsuited for, e.g., on-line monitoring of DST in washing machines.

In this paper, we propose a new method of DST determination. related to the pressure-jump method. Instead of the conventional sparging, we use the (cathodic) electrolysis of water at an actuator to produce gas bubbles in situ [1,4].

For proper operation, it is necessary to have a reproducible single gas nucleation site. We propose the integration of a small nucleation core, electrolytically filled with gas, forming a Harvey nucleus on which the subsequent formation of a single gas bubble is energetically favoured. Thus, we constructed a gas phase nucleation core electrode (GPNC) depicted in fig. 1. An additional and important advantage of the device is that the actuator can also function as the sensor for the bubble frequency by monitoring either its overpotential or its impedance. Both signals contain the periodic information caused by the bubble detachment. A block diagram, showing the sub-processes of our proposed method of DST determination, including some relevant input and output parameters, is shown in fig. 2 .

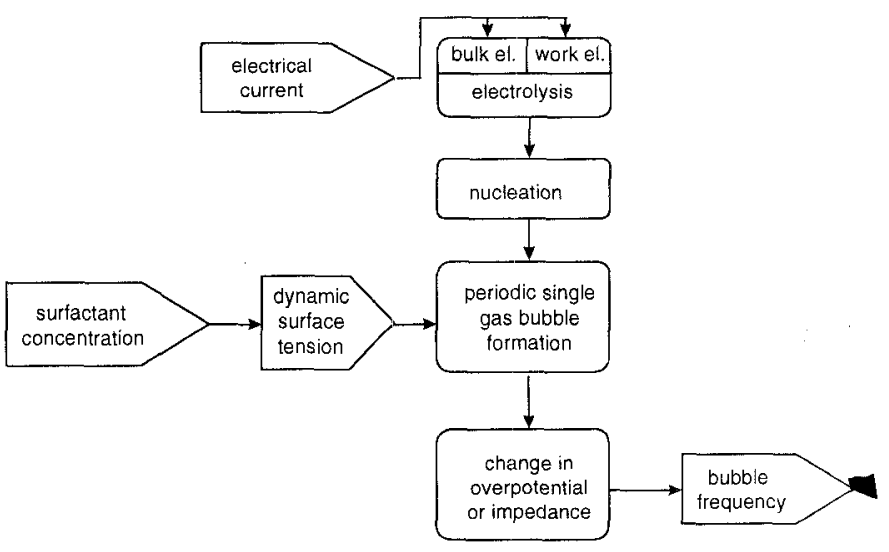

Fig. 2 Block diagram showing the sub-processes of the proposed method of DST determination with relevant input and output parameters. 


\section{THEORY}

The cathodic electrolysis of water at an actuator is used to produce gas bubbles:

$$
\mathrm{H}_{2} \mathrm{O}+4 e^{-} \rightarrow 4 \mathrm{OH}^{-}+2 \mathrm{H}_{2}
$$

A simplified expression for the gas production rate $Q_{g}$ is

$$
Q_{g}=\frac{i}{z F} V_{m}
$$

where $i$ is the electrolysis current, $F$ the Faraday, $z$ the number of electrons involved in the hydrogen evolution reaction: $z=2$, and $V_{m}$ the molar volume (being a constant at fixed pressure). Assuming a bubble volume $V$ at the moment of detachment from the actuator, the bubble frequency then becomes

$$
v=\frac{Q_{g}}{V}
$$

Assuming a horizontal actuator and with some additional simplifications, an equilibrium exists at the moment of detachment between the detaching force, being the buoyancy only, and the attaching force, containing the surface tension of the liquid:

$$
V\left(\rho_{1}-\rho_{2}\right) g=\pi \phi \sigma
$$

with $\rho_{l}, \rho_{2}$ the densities of the hydrogen gas and the liquid, respectively, $g$ the gravitational acceleration, $\phi$ the diameter of the gas bubble at the actuator, and $\sigma$ the surface tension of the liquid. The combination of eqns. $2-4$ yields for the bubble frequency $v$

$$
\nu=\frac{g\left(\rho_{1}-\rho_{2}\right)}{\pi \phi \sigma} \cdot \frac{i V_{m}}{z F}=\mathrm{constant} \cdot \frac{i}{\sigma}
$$

The relation between the surface tension, $\sigma$, and the surfactant concentration is described by adsorption. The adsorption equilibrium is governed by two equations. Firstly, the Gibbs relation specifies the change of the equilibrium surface tension, $\sigma$, due to the adsorption of surfactant at the surface [5]:

$$
d \sigma=-\Gamma d \mu=-\frac{\Gamma R T}{c_{0}} \cdot d c_{0}
$$

where $\Gamma\left[\mathrm{mol} / \mathrm{m}^{2}\right]$ is the surface density of the surfactant, $\mu$ is the chemical potential of the surfactant $[\mathrm{J} / \mathrm{mol}], \mathrm{R}$ is the gas constant $[\mathrm{J} /(\mathrm{mol} \cdot \mathrm{K})], T$ is the absolute temperature $[\mathrm{K}]$, and $\mathrm{c}_{0}$ is the concentration of the surfactant in the subsurface layer $\left[\mathrm{mol} / \mathrm{m}^{3}\right]$. The subsurface is defined as being a film that directly borders the surface. At equilibrium, the subsurface concentration is also the bulk concentration of the surfactant.

Secondly, an equation is needed to relate the surfactant concentration in the subsurface layer, $c_{0}$, to the surface density,

$\Gamma$, hence an adsorption isotherm. Many nonionic surfactants are found to satisfy the empirical Langmuir adsorption isotherm [6]

$$
\Gamma=\Gamma_{\infty} \frac{c_{0}}{c_{0}+C_{1 / 2}}
$$

where $\Gamma_{\infty}$ is the saturation surface density $\left[\mathrm{mol} / \mathrm{m}^{2}\right]$ and $C_{1 / 2}$ is the concentration at which the equilibrium surface density becomes half its saturation value. $\Gamma_{\infty}$ is an interface specific parameter. A characteristic of this equation is the fact that $\Gamma=\Gamma_{\infty}$ only if $\mathrm{c}_{0}>\mathrm{C}_{1 / 2}$.

The combination of eqns. (6) and (7) yields after integration a relation between the surface tension and the surfactant concentration (the Szyszkowski equation) [7]:

$$
\sigma-\sigma_{0}=-\Gamma_{\infty} R T \cdot \ln \left(\frac{c_{0}}{C_{1 / 2}}+1\right)
$$

where $\sigma_{0}$ is the surface tension of the solvent (water in this case). Eqn. (8) predicts a decreasing surface tension with increasing surfactant concentration, $c_{0}$, and with increasing temperature, $\mathrm{T}$.

Essentially, two processes take place to reach equilibrium in a surfactant solution, not only the adsorption at the surface as described above, but also the transport of surfactant to the surface. If the surfactant adsorption process is fast compared to the transport, the concentration in the subsurface layer is in equilibrium with the surface density and there is a concentration gradient from the bulk towards the subsurface layer. Under these conditions, the relations for adsorption equilibrium are still valid, but for a time dependent concentration in the subsurface layer, $c_{0}(t)$. The treatment of these time-dependent coupled processes is beyond the scope of this paper.

\section{EXPERIMENTAL}

\section{Device preparation}

The gas phase nucleation core electrodes (fig. 1) were made in silicon technology. Silicon wafers with a $<100>$ crystal orientation were used. The cavities were isotropically etched using reactive ion etching in silicon through a $\mathrm{SiO}_{2}$ mask (insulator layer in fig. 1). Devices with mouth diameters of 2, 3, 5 , or $10 \mu \mathrm{m}$ were fabricated.

A sandwich of $\mathrm{Ti} / \mathrm{Au} / \mathrm{Ti}$ with a thickness of $25 / 250 / 25 \mathrm{~nm}$, respectively, was evaporated and patterned on the wafers. The active area of the electrodes was defined by a passivation layer consisting of a sandwich of $\mathrm{SiO}_{2}, \mathrm{Si}_{3} \mathrm{~N}_{4}$, and photocurable polyimide. The $\mathrm{SiO}_{2}, \mathrm{Si}_{3} \mathrm{~N}_{4}$ interlayers were included as a passivation for the polyimide layer, which otherwise can undergo redox reactions. At the end of this patterning process, the titanium top-layer was removed in the active area, allowing the gold surface a free contact with the aqueous solution. The active area of the microelectrodes used during the experiments was determined by the $\mathrm{SiO}_{2} / \mathrm{Si}_{3} \mathrm{~N}_{4} /$ polyimide window to a circle with the diameter of $35 \mu \mathrm{m}$. The wafers were then cut 
into pieces and the chips were glued on $8 \times 100 \mathrm{~mm}^{2}$ printed circuit board carriers. The metal strip was wire bonded and the silicon bulk was contacted with silver glue. The devices were then encapsulated by hand with Hysol resin.

\section{Measurement set-up}

A schematic of the complete measurement set-up used for the electrical monitoring of the gas bubbles is shown in figure 3 .

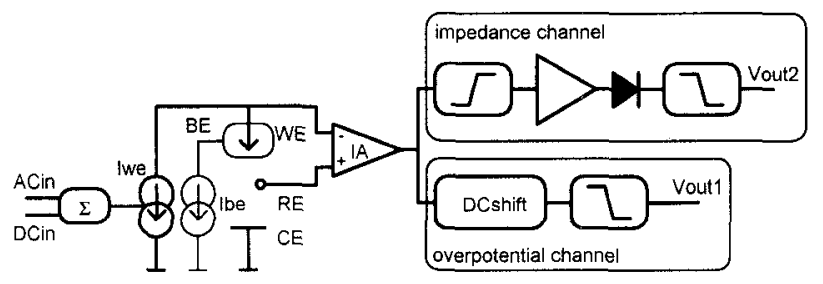

Fig. 3 Measurement set-up. WE is the working electrode, $C E$ is the counter electrode, $R E$ is the reference electrode, $B E$ is the bulk electrode. $I_{\text {we }}$ is the electrolysis current used to drive the working electrode and $I_{b e}$ is the current used for the generation of the gas phase nucleation core. ACin and DCin are the control voltages applied to the current source by means of the summing amplifier $\Sigma$. IA is the instrumentation amplifier. $v_{\text {out } 1}$ corresponds to the measured overpotential and $v_{\text {out } 2}$ corresponds to the measured impedance fluctuations.

With this specially designed set-up overpotential and impedance fluctuations due to bubble evolution could be measured simultaneously. The overpotential channel automatically removed the mean value of the measured potential, making it possible to monitor only the absolute value of the fluctuations around this mean value. For the impedance measurements, a signal (ACin) with a frequency of $100 \mathrm{kHz}$ and a small amplitude (typically $50 \mathrm{mVpp}$ ) was added to the control voltage of the current source (DCin) by means of a summing amplifier, $\Sigma$. The outputs of the two channels ( $V_{\text {out1 }}$ and $V_{\text {out2 }}$ ) were monitored and recorded on a dual channel digital oscilloscope. The bubble frequency was estimated from these recordings.

The experiments were done in a $10 \mathrm{mM}$ solution of sodium acetate (NaAc, Merck, analytical grade), at different concentrations of nonionic surfactant (Novel EO 4.5, courtesy of Unilever). Where needed, the $\mathrm{pH}$ was adjusted by adding small amounts of sodium hydroxide $(\mathrm{NaOH}$, Merck, analytical grade).

\section{RESULTS and DISCUSSION}

The need for the initial creation of a gas phase nucleus for proper operation of the device (i.e. with stable and periodic bubble evolution) was experimentally verified by testing the device deliberately before the formation of a gas phase nucleus. The reproducibility of the bubble evolution turned out to be very bad, without showing a constant periodicity. By optical observation of the gas evolution process it could be seen that several bubbles evolved at the same time, even from this small electrode surface $\left(35 \times 35 \mu \mathrm{m}^{2}\right)$. It can be concluded that the reproducibility of the gas bubble evolution process is not good enough in case the gas phase nucleus is not previously generated in-situ. The reproducibility was improved when the gas phase nucleus was generated from the bulk silicon electrode by means of a small constant current $I_{\text {be }}$. A typical experiment was carried out at a fixed surfactant concentration to measure the frequency and determine its stability. The result is shown in fig. 4 .

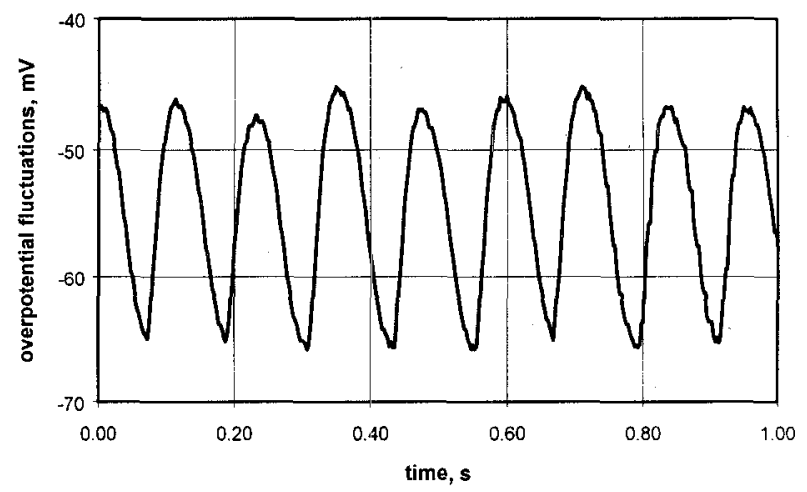

Fig. 4 Constant frequency during the bubble evolution from $G P N C$ electrodes illustrated by means of the recording of the overpotential fluctuations. Measurement in $10 \mathrm{mM} \mathrm{NaAc}$ with $0.25 \mathrm{~g} / \mathrm{l}$ Novel EO4.5 surfactant (courtesy of Unilever). The estimated frequency is $8.25 \mathrm{~Hz}$. The experiment was performed at $I_{w e}=I \mu \mathrm{A}$ and $I_{b e}=0.8 \mu \mathrm{A}$. The origin of the overpotential fluctuations is arbitrary.

The periodicity of the signal is obvious. It can be deduced from this periodicity that indeed the gas generated with the gold top electrode diffuses to the already existing gas phase in the nucleation core leading to a growing bubble rather than nucleating an independent bubble.

Finally, the effect of the surfactant concentration on the bubble frequency is presented in fig. 5 for a constant electrolysis current.

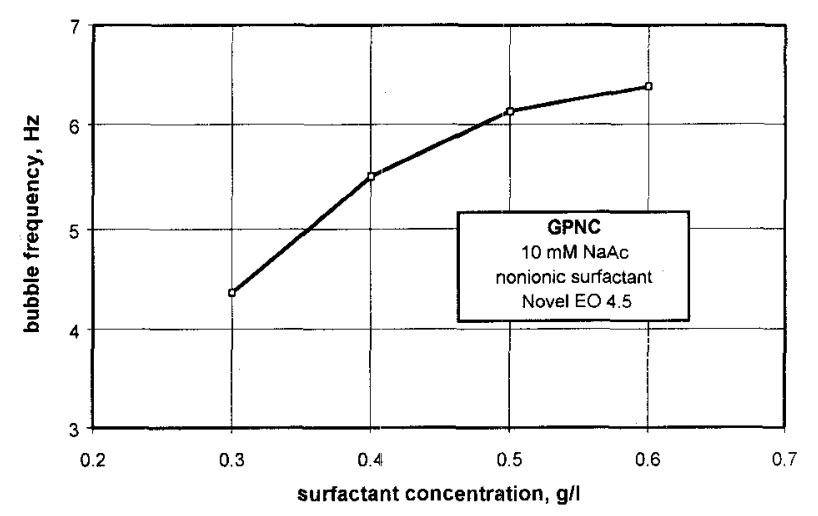

Fig. 5 Dependency of the bubble frequency on the surfactan concentration. $p H=11.38 . I_{w e}=0.6 \mu \mathrm{A} . I_{b e}=0 \mu \mathrm{A}$ (see text). 
Note that $I_{b e}=0 \mu A$ during the measurements, but the nucleation core was filled with gas by electrolysis just before the experiments by means of a small current of $\approx 1 \mu \mathrm{A}$ for a number of seconds. At a constant current of $\mathrm{I}_{\mathrm{we}}=0.6 \mu \mathrm{A}$ through the working electrode, an increase of the bubble frequency is observed for a higher surfactant concentration and thus for a lower dynamic surface tension. This increase in the bubble frequency was expected from eqn. 5 because by increasing the surfactant concentration, the surface tension of the solution decreases, according to eqn. 8 and the attachment force that keeps the bubble on the electrode becomes smaller, according to eqn. 4 . It can be concluded from fig. 5 that the GPNC electrodes can be used as sensors for measuring the surface tension in aqueous solutions.

\section{CONCLUSIONS}

We have proposed a new method of DST determination, using electrolysis of water at an actuator to produce gas bubbles in situ.

It has been shown that a gas nucleation site is necessary for proper operation. This site, guaranteeing single bubble generation, is accomplished by integrating a small nucleation core, electrolytically filled with gas.

The actuator also functions as the sensor for the detection of the bubble frequency by monitoring its overpotential. Similar data can be obtained by monitoring its impedance [4].

Simplified equations are derived, indicating the relation between the bubble frequency and the surface tension, as well as the relation between the surface tension and the surfactant concentration.

Measurement results show the expected monotonous relation of the bubble frequency as a function of the surfactant concentration.

\section{References}

[1] A. Volanschi, W. Olthuis, R.T.J.M. van der Heijden and P. Bergveld, European Patent 94203609.6, 13-121994.

[2] T. Müller-Kirschbaum and E.J. Smulders, SOFW Journal, 118-7, (1992) 427.

[3] S.G. Oh, S.P. Klein and D.O. Shah, AIChE Journal, 38-1, (1992) 149.

[4] A. Volanschi, W. Olthuis and P. Bergveld, Sensors and Actuators A, Physical, 52 (1996) 18-22.

[5] C.J. van Oss, Interfacial forces in aqueous media, ed. Marcel Dekker, Inc. (1994) 14 and 299.

[6] P.A. Kralchevsky, Y.S. Radkov and N.D. Denkov, Journal of Colloid and Interface Science, 161 (1993) $361-365$ 